\title{
Preclinical safety evaluation of hepatic arterial infusion of oncolytic poxvirus
}

This article was published in the following Dove Press journal:

Drug Design, Development and Therapy

\author{
Euna Cho ${ }^{1,2, *}$ \\ Eun Jin Ryu $u^{2,3, *}$ \\ Fen Jiang 2,4 \\ Ung Bae Jeon ${ }^{3}$ \\ Mong Cho',2 \\ Cy Hyun Kim' \\ Miyoung Kim' \\ Nam Deuk Kim ${ }^{5}$ \\ Tae-Ho Hwang ${ }^{1,2}$ \\ 'Department of Pharmacology and \\ Medical Research Center (MRC), \\ Pusan National University School of \\ Medicine, Yangsan, Korea; ${ }^{2}$ Department \\ of Research and Development, \\ Bionoxx Inc, Seongnam-si, Korea; \\ ${ }^{3}$ Department of Radiology, Pusan \\ National University Yangsan \\ Hospital, Yangsan, Korea; ${ }^{4}$ School of \\ Pharmaceutical Science (Shenzhen), \\ Sun Yat-sen University, Guangzhou, \\ China; ${ }^{5}$ Department of Pharmacy and \\ Pusan Cancer Research Center, Pusan \\ National University, Busan, Korea \\ *These authors contributed equally \\ to this work
}

Correspondence: Tae-Ho Hwang Department of Pharmacology and Medical Research Center (MRC), Pusan National University School of Medicine, 20 Geumo-ro, Mulgeum-eup, Yangsan

50612 , Korea

$\mathrm{Tel}+82515107551$

Fax +82 5I 5107558

Email thhwang@pusan.ac.kr
Purpose: Oncolytic poxvirus has shown promise in treating various solid tumors, such as liver cancer, and administration of oncolytic poxvirus via the hepatic artery may provide more survival benefits than other routes of administration. However, there is a lack of safety information to guide the application of hepatic arterial infusion (HAI) of oncolytic poxvirus in human studies. To investigate the acute and chronic toxicity of HAI administration of oncolytic poxvirus in animals and provide safety information for future human studies.

Methods: $\mathrm{VV}^{\text {tk-}}$, a vaccinia poxvirus with inactivated thymidine kinase gene, was administered via HAI to rabbits with normal liver function under angiography $\left(1 \times 10^{8}\right.$ or $\left.1 \times 10^{9} \mathrm{pfu}\right)$, and rats with N-nitrosomorpholine-induced precancerous liver cirrhosis under open surgery $\left(1 \times 10^{8} \mathrm{pfu}\right)$. Body weights and survival were monitored and blood samples were collected for hematological and biochemical tests. Distribution of A56 (a specific marker for poxvirus infection) in rabbit organs was evaluated using immunofluorescence assays.

Results: HAI of high doses of $\mathrm{VV}^{\text {tk- }}$ did not cause any acute or chronic changes in body weight, survival or in biochemical, hematological tests in the 2 animal models, and none of the changes showed dose dependency (in rabbit study), or were influenced by liver cirrhosis (in rat study). A56 was not detected in any of the major rabbit organs.

Conclusion: HAI may provide a safe alternative route of oncolytic poxvirus administration for human studies.

Keywords: oncolytic virus, transhepatic angiography, hepatocellular carcinoma, liver cirrhosis

\section{Introduction}

Hepatocellular carcinoma (HCC) is one of the leading causes of cancer death worldwide. ${ }^{1}$ Around $70 \%$ patients of $\mathrm{HCC}$ are diagnosed at an advanced stage of the disease, and are not eligible for curative treatments, such as liver resection, ablation, and transplantation. The median overall survival of advanced HCC without curative treatment is between 4 and 10 months. ${ }^{2}$ There are currently limited available treatments for advanced HCC and their survival benefits remain unsatisfactory., ${ }^{3,4}$

Oncolytic poxvirus belongs to the oncolytic virus (OV) family. OVs are an emerging class of cancer therapeutics that have shown great promise in treating HCC. ${ }^{5} \mathrm{OVs}$ are engineered to replicate selectively in and destroy cancer cells by multiple mechanisms of action. ${ }^{6}$ Currently, the choice of route of administration for oncolytic poxvirus delivery is limited to intratumoral (IT) and intravenous (IV) injections. ${ }^{7,8}$ The efficacy of IV injection is often compromised by neutralizing antibodies in the circulation, ${ }^{9}$ and IT injection is superior to IV injection in terms of targeting disease but IT injection has limited value when tumors are inaccessible or scattered. Hepatic arterial infusion (HAI) is a routine practice for transarterial chemo-embolization and transarterial chemotherapy, and the recently US Food and Drug Administration (FDA)-approved 
transarterial radioembolization requires HAI administration as well. A large retrospective study conducted among patients with colorectal cancer and liver metastases shows that chemotherapy administered via HAI resulted in almost 2 years longer survival than chemotherapy administered via other routes. ${ }^{10}$ The benefit of HAI administration is impressive because HAI can introduce high doses of therapeutic agents directly to multiple tumor masses while keeping drugs' systemic toxicity to a minimum. HAI may become an attractive route of administration for OVs. However, the safety of HAI administration of OVs is of great concern, particularly after the tragic incident of a fatal systemic inflammatory response syndrome in a patient following adenoviral gene transfer. ${ }^{11}$ There have been a few preclinical and clinical studies on the safety of HAI of OVs, such as adenovirus, herpes simplex virus-1, and vesicular stomatitis virus (VSV), ${ }^{12-14}$ but there is no safety information for the HAI administration of oncolytic poxvirus. In addition, it should be noted that $>70 \% \mathrm{HCC}$ patients have liver cirrhosis and/or other liver disorders; in a previous study conducted by Altomonte et al, the HAI administration of VSV showed no acute (up to 3 days) toxicity in rats with liver fibrosis. ${ }^{15}$ In this study, we aimed to investigate the acute and chronic (up to 2-6 months) toxicity of HAI administration of oncolytic poxvirus in a rabbit model with normal liver function and in a rat model with N-nitrosomorpholin (NNM)-induced precancerous liver cirrhosis.

\section{Materials and methods Virus}

Oncolytic poxvirus with inactivation of thymidine kinase gene, was engineered from a Wyeth strain vaccinia virus (New York City Department of Health Laboratories) as described previously. ${ }^{16} \mathrm{VV}^{\mathrm{tk}-}$ was re-suspended in $0.1 \mathrm{~mL}$ of PBS for use. The injected virus doses in rabbit study were $1 \times 10^{8}$ and $1 \times 10^{9} \mathrm{pfu}$ and in rat study $1 \times 10^{8} \mathrm{pfu}$.

\section{Rabbit study}

Eight New Zealand white rabbits (Samtako, Osan, Korea) with an average weight of $3 \mathrm{~kg}$ were randomly assigned to a low- $\left(1 \times 10^{8} \mathrm{pfu}\right)$ or a high-dose $\left(1 \times 10^{9} \mathrm{pfu}\right)$ group, 5 rabbits ( 2 were from the low-dose group) were followed up for 3 weeks, and the rest for 8 weeks. HAI was performed under fluoroscopic guidance (Integris H5000F, Philips, Bothell, WA, USA) via central auricular arteries as previously described. ${ }^{17}$ In brief, an 18-gauge angiocath needle was inserted into the rabbits' central auricular artery (of right or left side). And a 2.0-F microcatheter (Progreat, Terumo, Tokyo, Japan) and a 0.016-inch guidewire (Meister, Asahi Intecc Co., Ltd, Aichi, Japan) were introduced into the central auricular artery under fluoroscopic guidance by interventional radiologists (Figure 1). The guidewire was advanced carefully into the left hepatic artery through the descending thoracic aorta. When the microcatheter tip was placed in the left hepatic artery, contrast medium (Omnipaque, GE Healthcare, Seoul, Korea) was injected intra-arterially, followed by $\left(1 \times 10^{8}\right.$ or $\left.1 \times 10^{9} \mathrm{pfu}\right) \mathrm{VV}^{\mathrm{tk}-}$ and normal saline. Body weight was measured weekly until sacrifice, and blood samples were collected before injection and 1, 2, 3, and 8 weeks post injection for biochemical and hematological tests. All animal care, handling, and study procedures were in compliance with Institutional Animal Care and Use Committee (IACUC) guidelines from the Ministry of Food and Drug Safety. The animal study protocols were approved by the Pusan National University IACUC and performed at the Laboratory Animal Resource Center of Pusan National University, South Korea.

\section{Immunofluorescence assay}

Organs were harvested from all the rabbits after sacrifice and prepared as paraffin-embedded tissue slides. In addition, paraffin-embedded tissue slides of tumors (HT-29 and
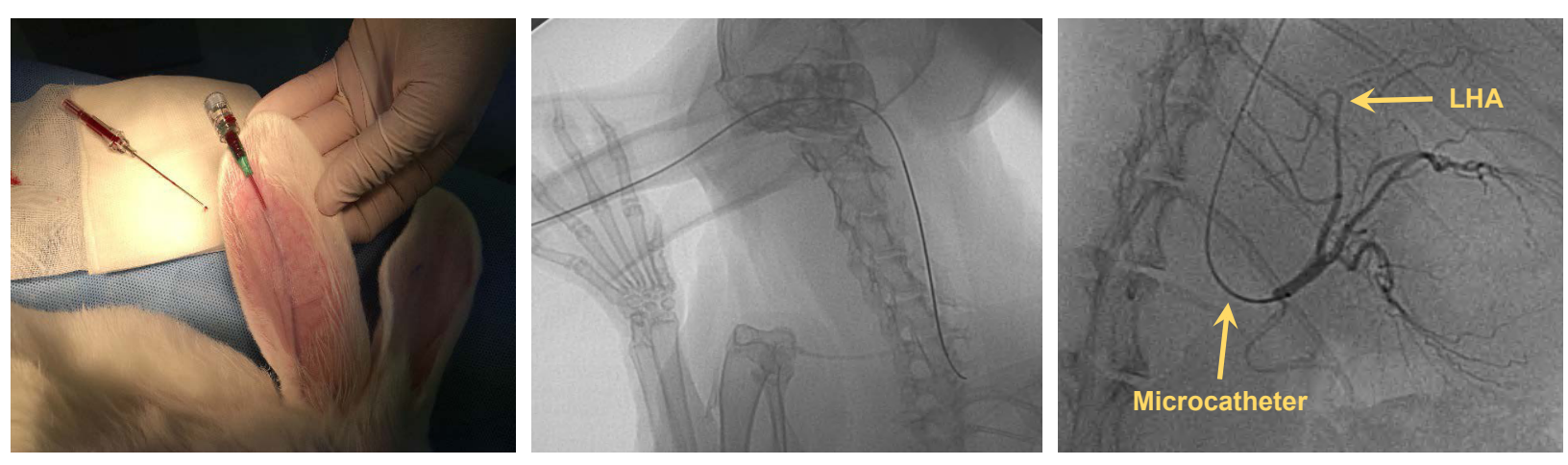

Figure I Selective hepatic artery angiography in a rabbit. Abbreviation: LHA, left hepatic artery. 
HCT-116) and non-tumor organs obtained from mice that received IT injections of $\mathrm{VV}^{\mathrm{tk}-}$ were used as positive and negative controls. The immunostaining was conducted using a M.O.M kit (FMK-2201, Vector, Burlingame, CA, USA), and the primary antibody was mouse anti-A56 monoclonal antibody (ABIN1606294, Antibodies-Online, Aachen, Germany), the secondary antibody was Alexa Fluor ${ }^{\circledR} 488$ antibody (A21204, Thermo Fisher Scientific, MA, USA). Fluorescence was monitored with an inverted research microscope (Eclipse Ti2, Nikon, Tokyo, Japan).

\section{NNM rat study}

NNM (Tokyo Chemical Industry Co., Ltd., Tokyo, Japan) was diluted in tap water. And 8-week old female Sprague-Dawley rats (Samtako, Osan, Korea) weighing 200-230 g were given $120 \mathrm{mg} / \mathrm{L} \mathrm{NNM}$ water for the first 4 weeks, and $200 \mathrm{mg} / \mathrm{L}$ for the following 4 weeks, and regular tap water for another 2 weeks. ${ }^{18}$ After the NNM pretreatment, 26 rats remained alive, and 2 of the rats were sacrificed for a liver histopathologic examination, and 24 rats were randomly assigned to a treatment group $(n=13)$ or a control group $(n=11)$, to receive the first HAI of $1 \times 10^{8} \mathrm{pfu} \mathrm{VV}^{\mathrm{tk}-}$ or PBS under an open abdomen surgery. ${ }^{19}$ The second HAI was given 24 weeks later for safety evaluation at a more advanced stage of the liver diseases because it is estimated that liver tumors develop between week 16 and 20 in this model, ${ }^{18}$ and 2 weeks later all the rats were sacrificed. Body weight was measured weekly until death or sacrifice. Because the open surgery may impose a huge impact on the biochemical tests, blood samples were not collected for the first HAI, instead, they were collected 1 and 3 days prior to the second HAI, and 1, 3, 5, and 7 days afterwards for aspartate aminotransferase (AST) and alanine transaminase (ALT) tests, using AST and ALT activity assay kits (colorimetric/fluorometric) (ab105135 and ab105134, Abcam, Cambridge, UK).

\section{Statistical analysis}

All data compared between 2 groups over different time points were analyzed using a linear mixed model analysis by SPSS software 21.0. A Kaplan-Meier survival curve was plotted and log-rank test was conducted using GraphPad Prism 5.03, a $P$-value $<0.05$ was considered statistically significant.

\section{Results}

\section{Toxicity of HAl administration of $\mathrm{V}^{\text {tk- }}$ in healthy rabbits}

No rabbits died between the HAI and the sacrifice day. One week after HAI of $\mathrm{VV}^{\text {tk-}}$, we observed an average of $230 \mathrm{~g}$ transient body weight decrease in all but 1 rabbit from the

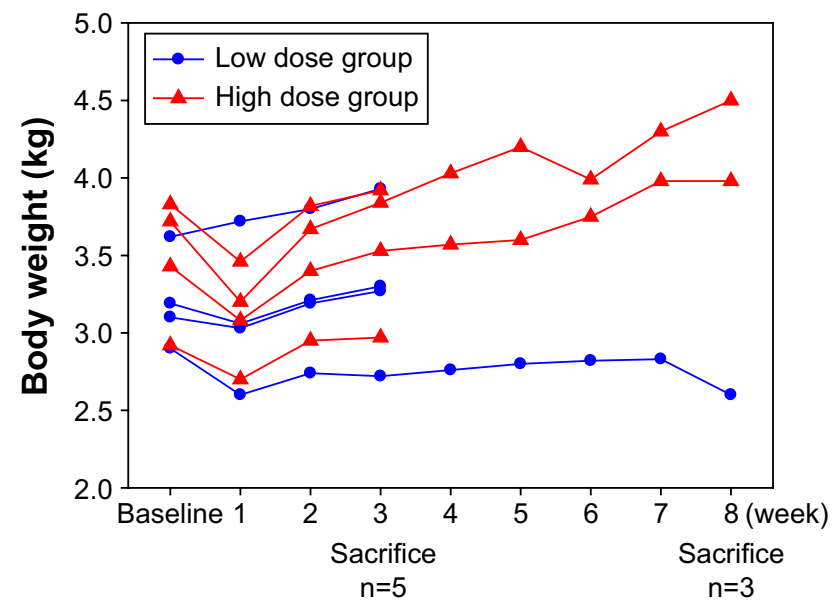

Figure 2 Body weight of 8 rabbits with normal liver function, measured at baseline and post hepatic artery infusion (week) of a single low $\left(I \times 10^{8}\right.$ pfu; $\left.n=4\right)$ and a high $\left(\mathrm{I} \times 10^{9} \mathrm{pfu} ; \mathrm{n}=4\right)$ dose of $\mathrm{VV}^{\mathrm{tk}-}$.

low-dose group (Figure 2). We also observed transient changes in the liver and renal functions in more than half of the rabbits, but these rabbits showed complete recovery 1 week later (Figure 3). In addition, the hematological changes were mild (Figure 4), and the changes in body weight, liver, and renal functions were not significantly different between the 2 dose groups, or between female and male rabbits.

\section{Expression of A56 in rabbit organs}

The immunofluorescence assay detected expression of A56 on the membrane of tumor cells, but not in other organs obtained from 2 different human tumor-bearing (HT-29 and HCT-116) mice that received IT injections of $\mathrm{VV}^{\mathrm{tk}-}$. In contrast, A56 was not detected in any other non-tumor organs in the mice, neither was A56 detected in the rabbit organs (Figure 5).

\section{Toxicity of $\mathrm{HAl}$ administration of $\mathrm{VV}^{\mathrm{t} k-}$ in NNM-induced liver cirrhotic rats}

Histopathological examinations confirmed the establishment of liver cirrhosis in the 2 rats sacrificed after the NNM pretreatment (Figure 6). Survival and body weight were monitored for 26 weeks after the first injection (Figure 6). Three of the 13 rats in the $\mathrm{VV}^{\text {tk- }}$ group and 3/11 in the PBS group died within 1 week after the first injection. After the second injection, $0 / 13$ and $1 / 11$ rats from the 2 groups, respectively, died within 1 week. By week 26, 5/13 rats in the $\mathrm{VV}^{\text {tk- }}$ group and 2/11 in the PBS group survived. There was no statistical difference in survival between the 2 groups. One week after the first injection, the mean body weights were $101.2 \%$ 

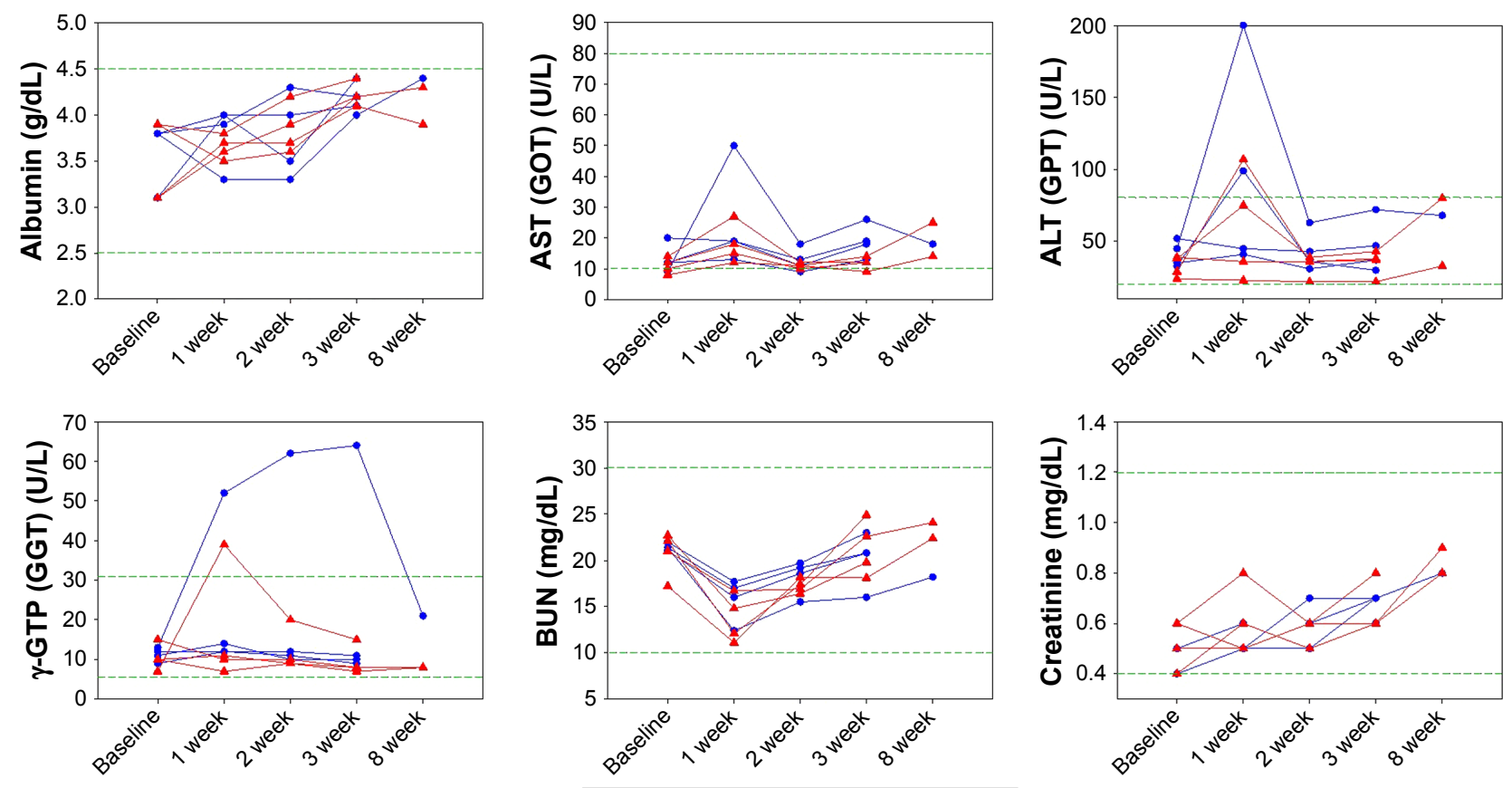

Figure 3 Organ functions of 8 rabbits with normal liver function, measured at baseline and post hepatic arterial infusion (week) of a single low (I $\times 10^{8}$ pfu; $\left.n=4\right)$ and high $\left(\mathrm{I} \times 10^{9} \mathrm{pfu} ; \mathrm{n}=4\right)$ dose of $\mathrm{VV}^{\mathrm{tk}-}$.

Note: Distances between 2 green dash lines are reference ranges.

Abbreviations: ALT, alanine transaminase; AST, aspartate aminotransferase; BUN, blood urea nitrogen; GGT, gamma-glutamyl transpeptidase; GOT, glutamic-oxalacetic transaminase; GPT, glutamic-pyruvic transaminase.
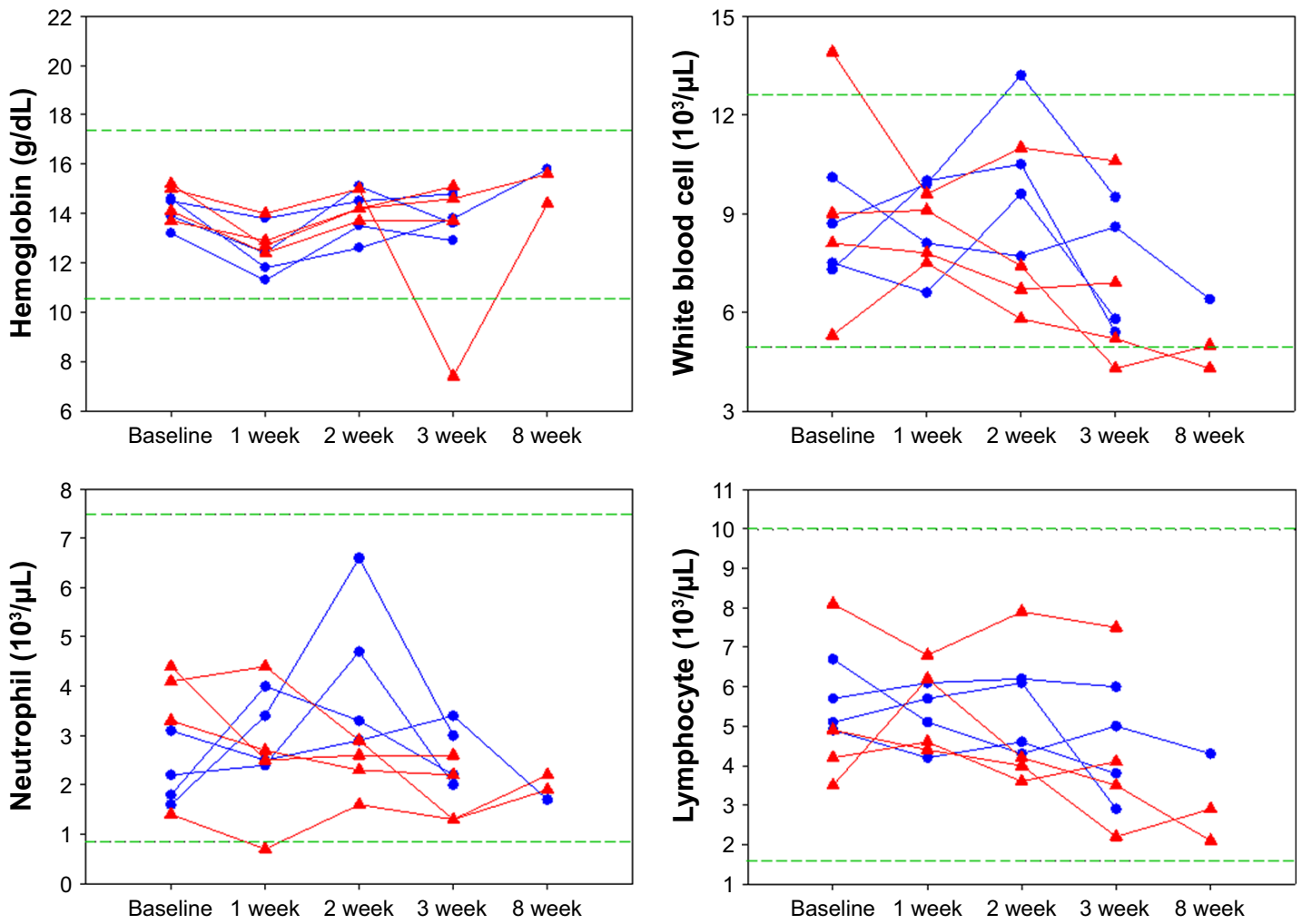

Low dose group $\rightarrow$ High dose group

Figure 4 Hematological tests of 8 rabbits with normal liver function, measured at baseline and post hepatic artery infusion (week) of a single low $\left(I \times I 0^{8}\right.$ pfu; $\left.n=4\right)$ and a high $\left(\mathrm{I} \times 10^{9}\right.$ pfu; $\left.\mathrm{n}=4\right)$ dose of $\mathrm{VV}^{\mathrm{tk}-}$.

Note: Distances between 2 green dash lines are reference ranges. 


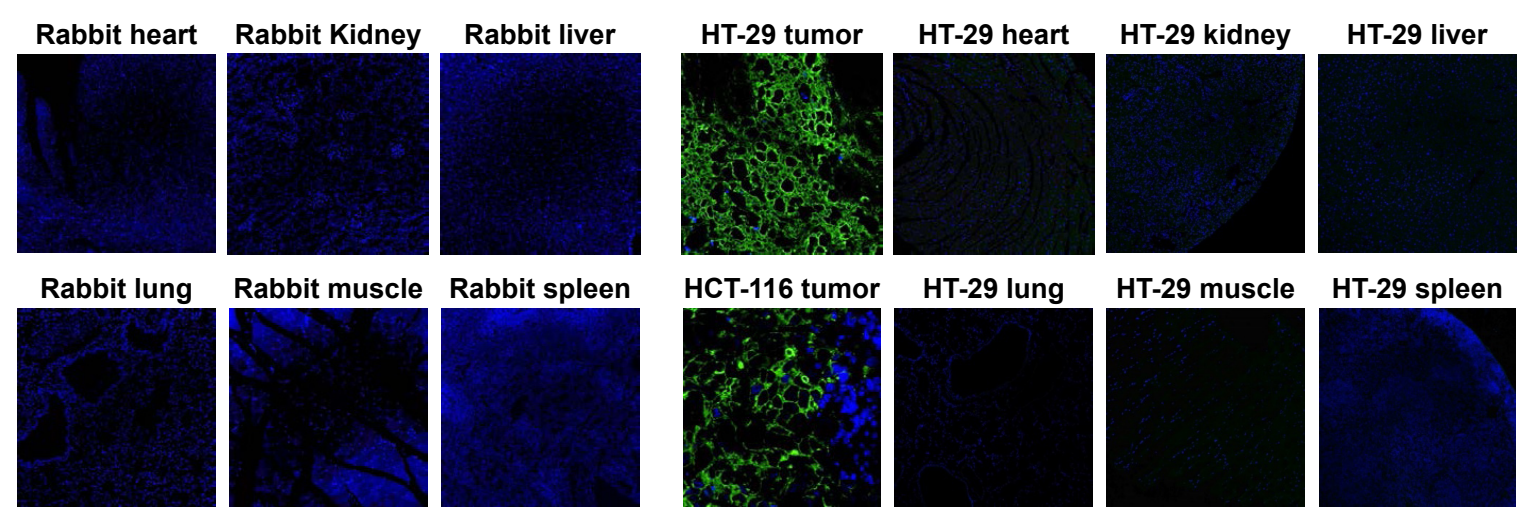

Figure 5 Immunofluorescence assay detected no A56 expression in multiple organs of rabbits with normal liver function, after hepatic artery infusion of a single dose $\left(\mathrm{I} \times 10^{9} \mathrm{pfu}\right)$ of $\mathrm{VV}^{\mathrm{tk}-}$.

Notes: The expression of A56 in tumor tissue and other organs from humanized tumor mouse models (HT-29 and HCT-II6) serves as positive and negative controls. Green: A56; blue: nuclei.

(\% of the baseline) for the $\mathrm{VV}^{\mathrm{tk}-}$ group and $95.6 \%$ for the PBS. Statistically, the body weight increase was slightly higher in the $\mathrm{VV}^{\mathrm{tk}-}$ group than in the PBS $(P<0.001)$. As the number of survival animals decreased, the body weight change became visually indistinguishable between the 2 groups. In addition, the AST levels measured before and after the second injections indicated no statistical difference between the 2 groups. In contrast, ALT levels were statistically lower in the $\mathrm{VV}^{\mathrm{tk}-}$ group than in the PBS group (Figure 7).

\section{Discussion}

To our knowledge, this is the first study that explored the safety of HAI administration of high doses of oncolytic poxvirus, particularly under conditions of liver cirrhosis. And it is the first study that used the expression of A56, an oncolytic poxvirus-specific membrane protein as a marker for preclinical safety evaluation.

It was estimated that the doses of oncolytic poxvirus administered to the 2 animal models were equivalent to $1 \times 10^{9}-1.5 \times 10^{10} \mathrm{pfu}$ in humans, higher than the maximum dose $\left(3 \times 10^{9} \mathrm{pfu}\right)$ of JX-594 (an oncolytic poxvirus) that was used in clinical trials. ${ }^{7}$ In the rabbit study, we observed transient changes in body weight and organ functions after the HAI of $\mathrm{VV}^{\mathrm{tk}-}$ in both high- and low-dose groups. However, these changes were unlikely due to the virus infection because none of the changes showed dose dependency. Instead, they were more likely physiological responses to the invasive procedure of HAI, as similar changes were observed in other HAI studies as well. ${ }^{20,21}$ In the rat study, the AST/ALT measured before and after the second HAI

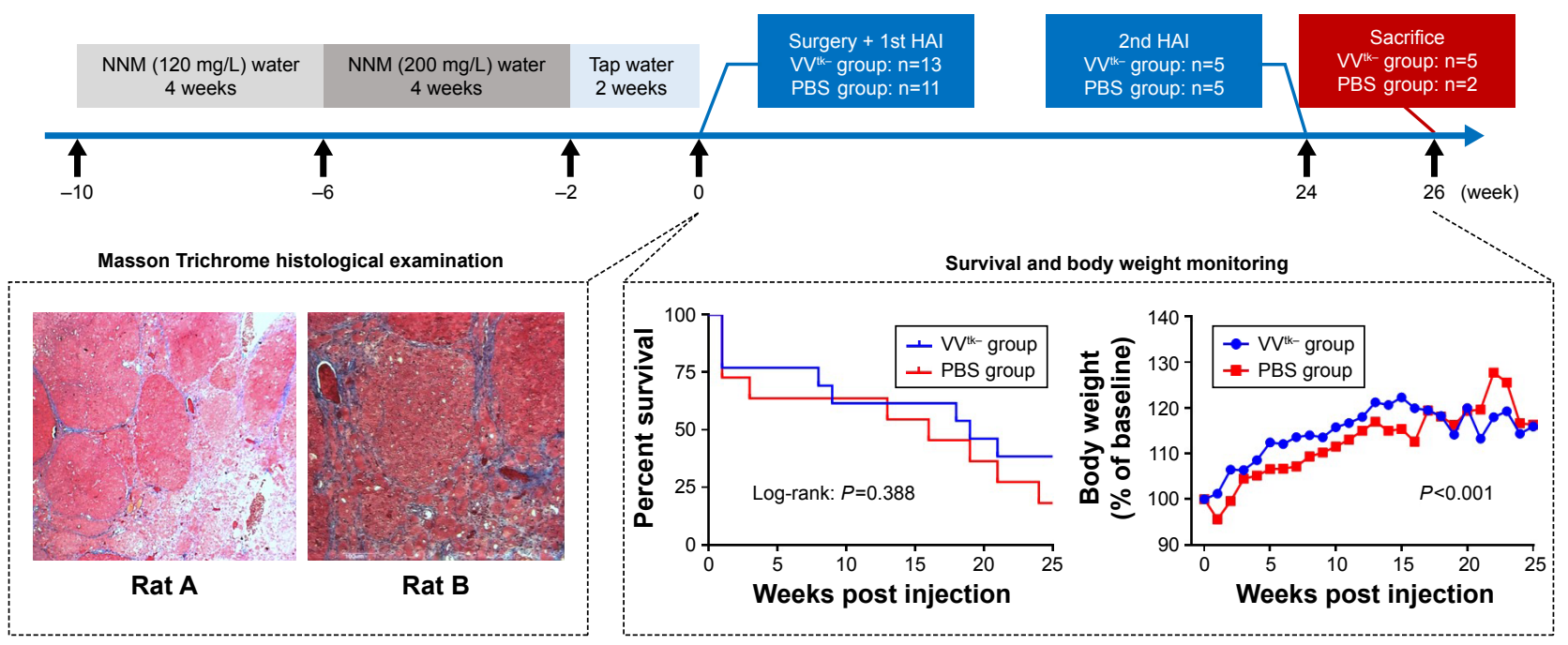

Figure 6 Study design (upper) and study results (lower) of the rat study. Masson Trichrome histological examination was conducted in liver tissue obtained from 2 rats (at week 0 ) that finished the NNM pretreatment, to confirm the formation of liver cirrhosis. Survival and body weight were monitored between week 0 and 26 . Survival was not significantly different $(P=0.388)$ between the 2 groups, $P$-value was obtained using log-rank test, and body weight increase (\% of baseline) was significantly higher in the $V^{\text {tk- }}$ group than the PBS one, $P$-value was obtained using linear mixed model analysis $(P<0.00 \mathrm{I})$.

Abbreviation: NNM, N-nitrosomorpholine. 


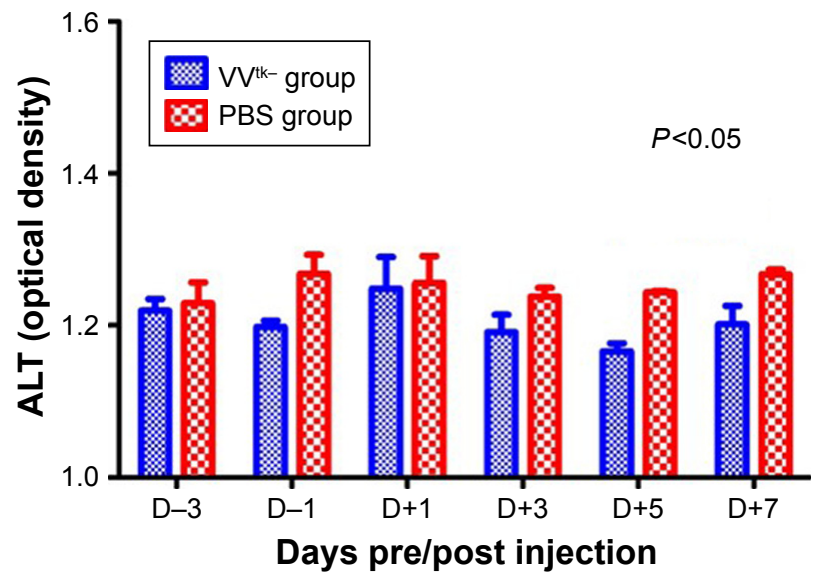

Figure 7 ALT measured before and after the second $\mathrm{HAI}\left(\mathrm{I} \times 10^{8}\right.$ pfu $\mathrm{VV}^{\text {tk- }}$ or PBS) in NNM-induced liver cirrhotic rats.

Note: Comparison between the 2 groups was conducted using linear mix model analysis.

Abbreviations: ALT, alanine transaminase; HAl, hepatic arterial infusion; NNM, N-nitrosomorpholin.

(the second HAI received little impact from surgery) were not different, which further proves that HAI of $\mathrm{VV}^{\text {tk- }}$ is unlikely to cause any acute liver toxicity.

In vitro and in vivo markers that are commonly used for monitoring OVs include the following: 1) overall tumor genes' expression, 2) specific genes' expression in tumor cells, and 3) transgenes introduced into the viral genes. ${ }^{22}$ And the recently developed fourth class of markers are viral genes, such as A56 that was used in this study. A56 is expressed exclusively on the membrane of cells that are infected by poxvirus, ${ }^{23}$ and because oncolytic poxvirus $\mathrm{VV}^{\mathrm{tk}-}$ was designed to selectively infect cancer cells, in our study, A56 was only detected in tumor tissues but not in other non-tumor tissues after HAI administration of high doses of $\mathrm{VV}^{\mathrm{tk}-}$, suggesting that HAI is a safe route of administration with regards to organ accumulation and toxicity. In future preclinical studies, A56 can be particularly valuable in monitoring oncolytic poxvirus replication status in tumor tissues. A56 expression data together with the kinetic data (eg, viral load in the circulation) can be important in developing pharmacokinetic-pharmacodynamic models for oncolytic poxvirus. Notably, the positive and negative controls for A56 used in this study were obtained from humanized tumor mice but not from rabbits, however, we assume the detection of mammalian-expressed vaccinia virus protein A56 is unlikely influenced by animal species. ${ }^{24}$

NNM has been used to develop liver cirrhotic HCC animal models ${ }^{18}$ in this study, it was used to replicate chronic precancerous liver cirrhosis and its progression. The rats that received HAI of $\mathrm{VV}^{\mathrm{tk}-}$ did not demonstrate symptoms of acute toxicity (AST/ALT increase) or chronic toxicity (body weight decrease over 26 weeks). It should be noted that the AST/ALT tests were conducted after the second HAI and may have not accurately reflected the acute toxicity of the first HAI, because the $\mathrm{VV}^{\mathrm{tk}-}$ from the second HAI was likely to have been greatly eliminated by the anti-vaccinia neutralizing antibody that was developed after the first HAI. ${ }^{25}$ However, because 3 rats in both the $\mathrm{VV}^{\mathrm{tk}-}$ and PBS control groups died within a day after the first HAI, we speculated that the $\mathrm{VV}^{\mathrm{tk}-}$ treatment was not more toxic than the PBS control, and these deaths were likely due to the burden of the open surgery that was conducted at the first HAI. From over a week after the first injection to the end of the study, more than half of the rats died from both groups, likely due to progression of disease. However, we did not observe more toxicity or deaths in the $\mathrm{VV}^{\mathrm{tk}-}$ group, on the contrary, the overall survival in the $\mathrm{VV}^{\mathrm{tk}-}$ group (5/13) seems to be superior to the PBS control group (2/11) although there is no statistical significance (log rank: $P=0.388$ ). The non-significance is
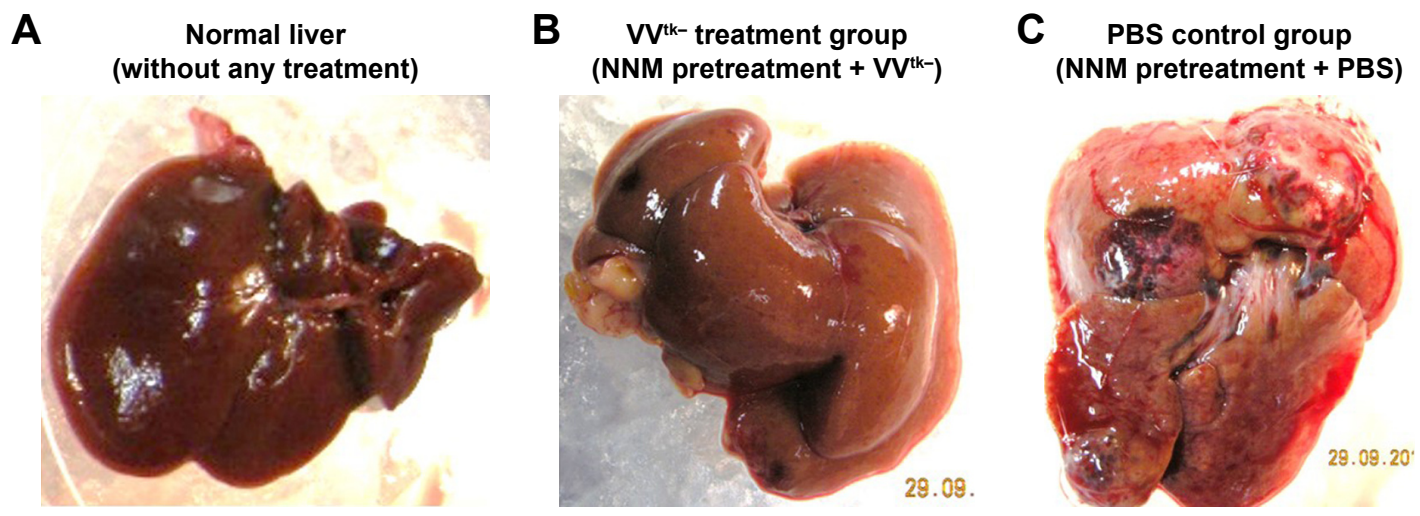

Figure 8 Liver tissue obtained from $(\mathbf{A})$ rats without any treatment, $(\mathbf{B})$ rats with NNM-induced precancerous liver cirrhosis after the second $\mathrm{HAI}$ of $\mathrm{I} \times \mathrm{I} 0^{8}$ pfu $\mathrm{V} \mathrm{V}^{\mathrm{tk}-}$, and (C) rats with NNM-induced precancerous liver cirrhosis after the second HAI of PBS.

Abbreviations: HAl, hepatic arterial infusion; NNM, N-nitrosomorpholin. 
probably because the sample sizes were too small and all the rats were sacrificed 2 weeks after the second HAI and there were not enough data to demonstrate any further survival benefit. What's more, the body weight changes and ALT tests were significantly more favorable in the $\mathrm{VV}^{\mathrm{tk}-}$ group than in the PBS $(P<0.05)$. Surprisingly, the livers obtained from sacrificed rats in the $\mathrm{VV}^{\text {tk- }}$ group looked more like normal livers than cirrhotic ones. In contrast, livers obtained from sacrificed rats in the PBS group had developed visible multiple tumors (Figure 8). Because it was not our primary objective to evaluate the treatment efficacy in this study, the rats were sacrificed after the second HAI administration and the rat organs were not harvested for histopathological examinations. We have conducted other studies to test the efficacy of $\mathrm{VV}^{\mathrm{tk}-}$ in preventing and reducing the progression of NNM-induced liver diseases, and will report the results soon.

\section{Conclusion}

Administration of high doses of oncolytic poxvirus via HAI may cause a transient liver function change (which is likely due to the HAI procedure), but is unlikely to cause any dosedependent or liver cirrhosis-related acute or chronic toxicity, neither will it cause accumulation of the virus in any nontumor organs. Therefore, we suggest that HAI of oncolytic poxvirus can be vastly explored for its therapeutic potency in HCC patients in future studies.

\section{Acknowledgments}

This study was supported by a grant from the National R\&D Program for Cancer Control, Ministry of Health and Welfare, Republic of Korea (HA16C0013) and National Research Foundation of Korea (NRF) grant funded by the Korea government (MSIP) (NRF-2015R1A5A2009656).

\section{Author contributions}

Eun Jin Ryu and Tae-Ho Hwang designed the rabbit study. Eun Jin Ryu, Cy Hyun Kim, and Miyoung Kim conducted the rabbit study. Euna Cho and Mong Cho designed the rat study. Ung Bae Jeon conducted the angiography and Euna Cho conducted the rest of the rat study. All authors contributed toward data analysis, drafting and critically revising the paper, gave final approval of the version to be published, and agree to be accountable for all aspects of the work.

\section{Disclosure}

Part of the data in this study is going to be submitted for an investigational new drug application by Bionoxx Inc. The authors have no conflicts of interest to declare in this work.

\section{References}

1. Lozano R, Naghavi M, Foreman K, et al. Global and regional mortality from 235 causes of death for 20 age groups in 1990 and 2010: a systematic analysis for the Global Burden of Disease Study 2010. Lancet. 2012;380(9859):2095-2128.

2. Cabibbo G, Enea M, Attanasio M, Bruix J, Craxì A, Cammà C. A metaanalysis of survival rates of untreated patients in randomized clinical trials of hepatocellular carcinoma. Hepatology. 2010;51(4):1274-1283.

3. Cheng AL, Kang YK, Chen Z, et al. Efficacy and safety of sorafenib in patients in the Asia-Pacific region with advanced hepatocellular carcinoma: a phase III randomised, double-blind, placebo-controlled trial. Lancet Oncol. 2009;10(1):25-34.

4. Bruix J, Qin S, Merle P, et al. Regorafenib for patients with hepatocellular carcinoma who progressed on sorafenib treatment (RESORCE): a randomised, double-blind, placebo-controlled, phase 3 trial. Lancet. 2017;389(10064):56-66.

5. Jebar AH, Errington-Mais F, Vile RG, Selby PJ, Melcher AA, Griffin S. Progress in clinical oncolytic virus-based therapy for hepatocellular carcinoma. J Gen Virol. 2015;96(Pt 7):1533-1550.

6. Kirn D, Martuza RL, Zwiebel J. Replication-selective virotherapy for cancer: Biological principles, risk management and future directions. Nat Med. 2001;7(7):781-787.

7. Park BH, Hwang T, Liu TC, et al. Use of a targeted oncolytic poxvirus, JX-594, in patients with refractory primary or metastatic liver cancer: a phase I trial. Lancet Oncol. 2008;9(6):533-542.

8. Breitbach CJ, Burke J, Jonker D, et al. Intravenous delivery of a multimechanistic cancer-targeted oncolytic poxvirus in humans. Nature. 2011;477(7362):99-102.

9. Thorne SH, Liang W, Sampath P, et al. Targeting localized immune suppression within the tumor through repeat cycles of immune cell-oncolytic virus combination therapy. Mol Ther. 2010;18(9):1698-1705.

10. Groot Koerkamp B, Sadot E, Kemeny NE, et al. Perioperative Hepatic Arterial Infusion Pump Chemotherapy Is Associated With Longer Survival After Resection of Colorectal Liver Metastases: A Propensity Score Analysis. J Clin Oncol. 2017;35(17):1938-1944.

11. Raper SE, Chirmule N, Lee FS, et al. Fatal systemic inflammatory response syndrome in a ornithine transcarbamylase deficient patient following adenoviral gene transfer. Mol Genet Metab. 2003;80(1-2): $148-158$.

12. Carpenter SG, Carson J, Fong Y. Regional liver therapy using oncolytic virus to target hepatic colorectal metastases. Semin Oncol. 2010; 37(2):160-169.

13. Shinozaki K, Ebert O, Kournioti C, Tai YS, Woo SL. Oncolysis of multifocal hepatocellular carcinoma in the rat liver by hepatic artery infusion of vesicular stomatitis virus. Mol Ther. 2004;9(3):368-376.

14. Reid T, Galanis E, Abbruzzese J, et al. Hepatic arterial infusion of a replication-selective oncolytic adenovirus (d11520): phase II viral, immunologic, and clinical endpoints. Cancer Res. 2002;62(21):6070-6079.

15. Altomonte J, Marozin S, de Toni EN, et al. Antifibrotic properties of transarterial oncolytic VSV therapy for hepatocellular carcinoma in rats with thioacetamide-induced liver fibrosis. Mol Ther. 2013;21(11): 2032-2042.

16. Mackett M, Smith GL, Moss B. General method for production and selection of infectious vaccinia virus recombinants expressing foreign genes. J Virol. 1984;49(3):857-864.

17. Chang IS, Lee MW, Kim YI, et al. Comparison between transauricular and transfemoral arterial access for hepatic artery angiography in a rabbit model. J Vasc Interv Radiol. 2011;22(8):1181-1187.

18. Kim JH, Oh JY, Park BH, et al. Systemic armed oncolytic and immunologic therapy for cancer with JX-594, a targeted poxvirus expressing GM-CSF. Mol Ther. 2006;14(3):361-370.

19. van Etten B, Ten Hagen TL, de Vries MR, Ambagtsheer G, Huet T, Eggermont AM. Prerequisites for effective adenovirus mediated gene therapy of colorectal liver metastases in the rat using an intracellular neutralizing antibody fragment to p21-Ras. Br J Cancer. 2002;86(3): 436-442. 
20. Wang Y, Xiong B, Liang B, et al. Hepatic parenchymal changes following transcatheter embolization and chemoembolization in a rabbit tumor model. PLoS One. 2013;8(8):e70757.

21. Chan AO, Yuen MF, Hui CK, Tso WK, Lai CL. A prospective study regarding the complications of transcatheter intraarterial lipiodol chemoembolization in patients with hepatocellular carcinoma. Cancer. 2002;94(6):1747-1752.

22. Ansel A, Rosenzweig JP, Zisman PD, Gesundheit B. Monitoring the Efficacy of Oncolytic Viruses via Gene Expression. Front Oncol. 2017;7:264.

23. Dehaven BC, Gupta K, Isaacs SN. The vaccinia virus A56 protein: a multifunctional transmembrane glycoprotein that anchors two secreted viral proteins. J Gen Virol. 2011;92(Pt 9):1971-1980.
24. García AD, Meseda CA, Mayer AE, Kumar A, Merchlinsky M, Weir JP. Characterization and use of mammalian-expressed vaccinia virus extracellular membrane proteins for quantification of the humoral immune response to smallpox vaccines. Clin Vaccine Immunol. 2007;14(8):1032-1044.

25. Downs-Canner S, Guo ZS, Ravindranathan R, et al. Phase 1 Study of Intravenous Oncolytic Poxvirus (vvDD) in Patients With Advanced Solid Cancers. Mol Ther. 2016;24(8):1492-1501.

\section{Publish your work in this journal}

Drug Design, Development and Therapy is an international, peerreviewed open-access journal that spans the spectrum of drug design and development through to clinical applications. Clinical outcomes, patient safety, and programs for the development and effective, safe, and sustained use of medicines are the features of the journal, which has also been accepted for indexing on PubMed Central. The manuscript management system is completely online and includes a very quick and fair peer-review system, which is all easy to use. Visit http://www.dovepress.com/testimonials.php to read real quotes from published authors.

Submit your manuscript here: http://www.dovepress.com/drug-design-development-and-therapy-journal 